\title{
A RARE CASE OF CEFTRIAXONE INDUCED CORONARY SPASM WITH ACUTE MYOCARDIAL ISCHEMIA
}

Basavaraj G. T1 ${ }^{1}$ Ravikumar Ryakha² ${ }^{2}$ Sandeep ${ }^{3}$, Shreyas ${ }^{4}$

\section{HOW TO CITE THIS ARTICLE:}

Basavaraj G. T, Ravikumar Ryakha, Sandeep, Shreyas. "A Rare Case of Ceftriaxone Induced Coronary Spasm with Acute Myocardial Ischemia". Journal of Evolution of Medical and Dental Sciences 2014; Vol. 3, Issue 66, December 01; Page: 14414-14417, DOI: 10.14260/jemds/2014/3932

ABSTRACT: Kounis syndrome has been known as allergic angina and/or allergic myocardial infarction. Allergic insults usually may include drugs, latex, and food. Although ceftriaxone administration has been associated with various allergic reactions such as urticaria, angioedema, erythema, rash and anaphylactic shock, there are very few published report that has shown an association between ceftriaxone use and Kounis syndrome. Here, we describe a case report of allergic vasospasm, leading to acute anterior wall myocardial infarction, probably as the result of an acute allergic reaction, after ceftriaxone use.

KEYWORDS: ceftriaxone, allergy, acute coronary syndrome, kounis syndrome.

INTRODUCTION: Angina and acute myocardial infarction (AMI) secondary to allergy-induced coronary vasospasm are referred to as Kounis syndrome (KS). Many allergic factors including drugs, latex, food, contrast media have been implicated in causing KS. During allergic episodes, chemical agents such as histamine, leukotrienes, and neutral proteases are increased in the peripheral circulation, and hence, these agents may cause coronary artery spasm (CAS) and atheromatous plaque rupture.(1) Ceftriaxone is a third-generation cephalosporin antibiotic that has broad spectrum activity against Gram-negative and Gram-positive bacteria.(2) Although ceftriaxone administration has been associated with various allergic reactions such as urticaria, angioedema, erythema, rash and anaphylactic shock, $(2,3)$ there are very few case report about whether ceftriaxone gives rise to allergic acute coronary syndrome or not. Here, we define a case of severe CAS, leading to acute anterior myocardial infarction (MI), as the result of an acute allergic reaction, due to ceftriaxone use.

CASE REPORT: A 20-year-old male with no history of atopy or cardiovascular risk factors presented to our emergency department with fever and chills and burning micturition .Patient was admitted and given a $1 \mathrm{gm}$ i.v ceftriaxone after test dose. Approximately 5 minutes after the injection of ceftriaxone, patient complained of severe chest pain and sweating. On clinical examination, urticarial lesions with pruritis on the skin of the abdomen and legs were noted. His blood pressure was 80/50mmHg. Electrocardiogram (ECG) displayed sinus rhythm, heart rate of 60-65 bpm, and ST segment elevation on, Iand AVL (fig. -1) and ST depression with T wave inversion in lead II, III, and AVF. Compatible with acute anterior wall MI (Fig. 2). In the emergency department, for acute coronary syndrome, acetylsalicylic acid (300 mg), clopidogrel (a loading dose of $600 \mathrm{mg}$ ) and intravenous heparin (10,000 IU), IV nitroglycerine was administered. Following intravenous fluid, parenteral corticosteroids and antihistaminics were given for his allergic symptoms; his blood pressure increased to $110 / 70 \mathrm{mmHg}$, his urticarial lesions regressed, and AMI-related symptoms were improved over 4-6hrs. The patient underwent coronary angiography. Coronary angiogram was normal. First hour of admission, blood tests revealed CPK-MB was elevated and Troponin-T was positive. Twelve hours later, plasma CPK-MB value returned to normal range. 


\section{CASE REPORT}

Factor $\mathrm{V}$ Leiden and prothrombin gene mutation tests were executed and identified to be normal variant. Other tests disclosed the following: total cholesterol176 mg/dL (normal reference: 125-200), triglyceride $104 \mathrm{mg} / \mathrm{dL}$ (normal reference: 50-150), fibrinogen $2.6 \mathrm{~g} / \mathrm{L}($ normal reference: 2.0-4.5), homocysteine $5.2 \mu \mathrm{mol} / \mathrm{L}$ (normal reference: <12), antithrombin III activity 109\% (normal range: $70-125 \%$ ), prothrombin time 14 second (normal range: 10-14), and activated partial thromboplastin time 35second (normal range: 25-36). The patient was discharged without any complication after five days.

DISCUSSION: KS, defined as the co-incidental occurrence of acute coronary syndrome with hypersensitivity reactions following an allergic event, was first described by Kounis and Zavras in 1991 as an allergic angina syndrome.(4) Then, it was shown that KS is not only limited to anginal symptoms, but it also could progress to AMI. In addition, coronary vasospasm and/or plaque disruption can be provoked by allergic reactions on coronary vascular smooth muscle, resulting in a new cause of coronary vasospasm.(1) Several pathophysiologic mechanisms have been proposed to explain the cardiac involvement during anaphylactic reactions. ${ }^{(1,5)}$ Mast cell activation takes place by antigen antibody complex or the allergen itself following allergen exposure. Thereafter, several vasoconstricting and collagen degrading products, such as histamine, platelet activating factor, cytokines, chemokines, neutral proteases (tryptase, chymase), and renin are released into circulation. $(1,5,6)$

Moreover, the endothelial and platelet activation induced by histamine may further contribute to coronary plaque denudation and/or rupture, resulting in thrombus formation.(1) Hence, during an anaphylactic reaction, all of these factors may act together on the emergence of coronary vasospasm, as in the present case, eventually progressing to an AMI. There are two well-known variants of Kounis syndrome. The type-I variant is observed in patients with no cardiovascular risk factors and normal coronary arteries in whom the acute release of inflammatory mediators produces either CAS with normal cardiac enzymes and troponins or CAS leading to AMI accompained by elevated levels of markers of cardiac damage. The type-II variant includes patients with culprit but pre-existing atheromatous disease in whom acute hypersensitive reactions cause plaque erosion or rupture, culminating in AMI.(1) More recently, a type-III variant of KS has been defined in patients with a drug eluting coronary stent thrombosis.(1,7)

Some allergens have been implicated as the main trigger factors for KS. These allergens include drugs (antibiotics, analgesics, antineoplastics, thrombolytics, and others), contrast agent, latex, food.(1,5,7-9) Although there are many antibiotics that have been previously reported to cause KS. Ceftriaxone is rare cause.(8,9)

CONCLUSION: In conclusion, anaphylaxis by ceftriaxone is likely responsible for the severe CAS, leading in acute anterior wall MI in the present case. Complete recovery of clinical symptoms, hemotological, biochemical and angiographic findings show that KS caused by ceftriaxone should be kept in mind for the differential diagnosis of patients who presented with an AMI and allergic finding.

\section{REFERENCES:}

1. Kounis NG, Hahalis G, Kourelis T, Manola A, Theoarides TC. Kounis syndrome (allergic angina and allergic myocardial infarction). In: Angina Pectoris: Etiology, Pathogenesis and Treatment. Gallo AP, Jones ML, Eds. Nova Science Publishers, Hauppauge, 2008: 77-150. 


\section{CASE REPORT}

2. Bradley JS, Wassel RT, Lee L, Nambiar S. Intravenous ceftriaxone and calcium in the neonate: assessing the risk for cardiopulmonary adverse events. Pediatrics 123: e609-e613, 2009.

3. Romano A, Gaeta F, Valluzzi RL, Alonzi C, Viola M, Bousquet PJ. Diagnosing hypersensitivity reactions to cephalosporins in children. Pediatrics 122: 521-527, 2008.

4. Kounis NG, Zavras GM. Histamine-induced coronary artery spasm: the concept of allergic angina. Br J Clin Pract 45: 121- 128, 1991.

5. Fassio F, Almerigogna F. Kounis syndrome (allergic acute coronary syndrome): different views in allergologic and cardiologic literature. Intern Emerg Med 1-7, 2012 [Epub ahead of print].

6. Schwartz LB. Diagnostic value of tryptase in anaphylaxis and mastocytosis. Immunol Allergy Clin N Am 26: 451-463, 2006.

7. Akyel A, Murat SN, Cay S, Kurtul A, Ocek AH, Cankurt T. Late drug eluting stent thrombosis due to acemetacine: Type III Kounis syndrome Kounis syndrome due to Acemetacine. Int J Cardiol 155: 461-462, 2012.

8. Tavil Y, Turfan M, Turko_lu S, Abacı A. Kounis syndrome secondary to amoxicillin/clavulanic acid use. Int J Cardiol 124: e4-e7, 2008.

9. Calogiuri GF, Nettis E, Di Leo E, Vacca A, Ferrannini A, Kounis NG. Kounis syndrome induced by intravenous administration of piperacillin/tazobactam: A case report. Int J Cardiol 155: e42e44, 2012.

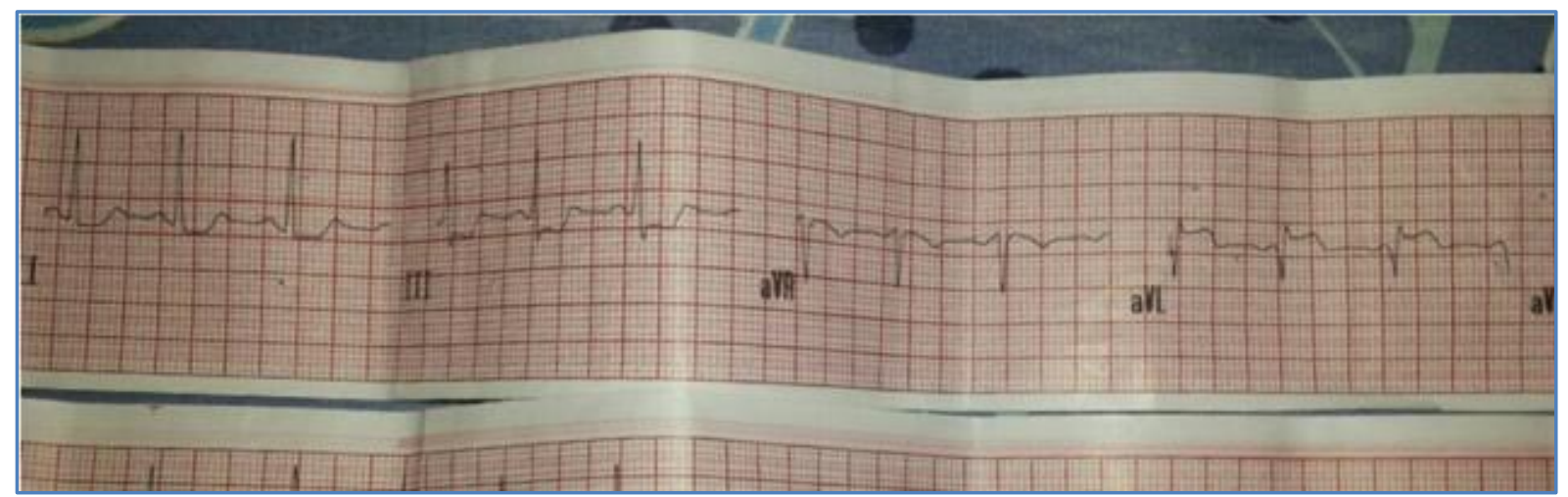

Fig. 1: S T segment ellevation in lead aVL and ST depression in lead III

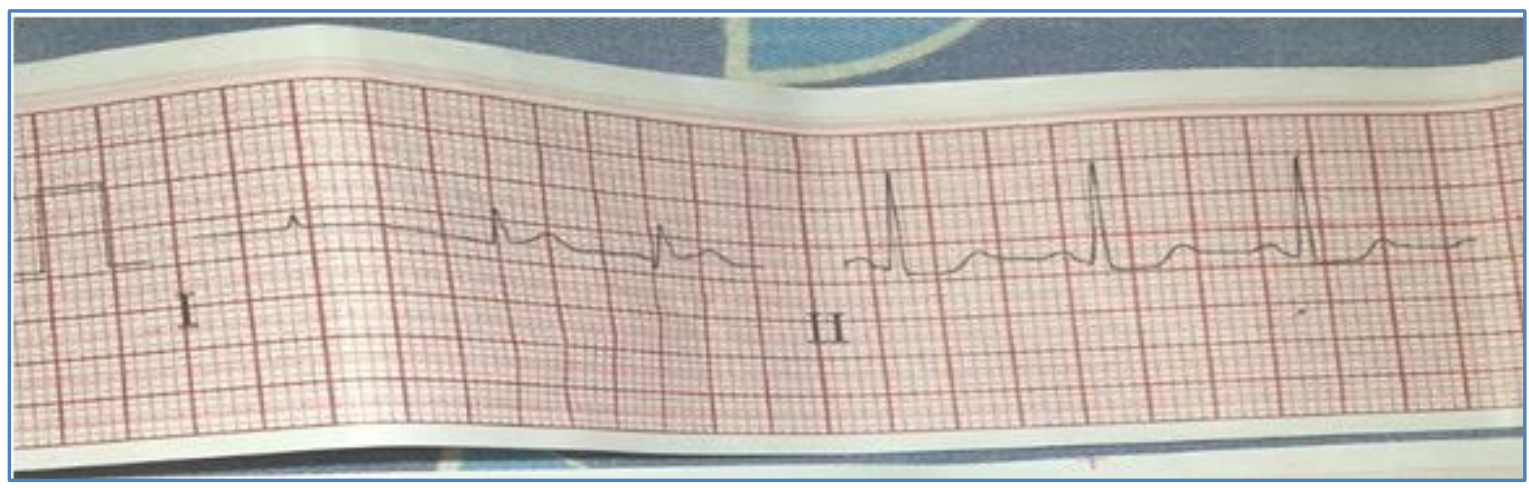

Fig. 2: ST segment elevation in lead I 


\section{AUTHORS:}

1. Basavaraj G. T.

2. Ravikumar Ryakha

3. Sandeep

4. Shreyas

\section{PARTICULARS OF CONTRIBUTORS:}

1. Senior Resident, Department of Medicine, Raja Rajeshwari Medical College and Hospital, Bangalore.

2. Senior Resident, Department of Medicine, Raja Rajeshwari Medical College and Hospital, Bangalore.

3. Post Graduate, Department of Medicine, Raja Rajeshwari Medical College and Hospital, Bangalore.
4. Post Graduate, Department of Medicine, Raja Rajeshwari Medical College and Hospital, Bangalore.

\section{NAME ADDRESS EMAIL ID OF THE CORRESPONDING AUTHOR:}

Dr. Ravikumar Ryakha, \#17, C- Block, $2^{\text {nd }}$ Floor, Moogambika Nivasa, Jayanna Layout, Near Raja Rajeshwari Temple,

R. R. Nagar, Bangalore.

Email: ryakha@gmail.com

Date of Submission: 20/11/2014.

Date of Peer Review: 21/11/2014.

Date of Acceptance: 27/11/2014.

Date of Publishing: 01/12/2014. 